Magda Lahorgue Nunes 1

Maurer Pereira Martins 1

Edmund Anthony Severn Nelson 2

Stephanie Cowan 3

Maria Luisa Cafferata 4

Jaderson Costa da Costa 1

\section{Orientações adotadas nas maternidades dos hospitais-escola do Brasil, sobre posição de dormir}

\author{
Instructions from teaching hospital maternity \\ wards to parents concerning the sleeping \\ position of newborns
}

1 Serviço de Neurologia, Hospital São Lucas, Pontifícia Universidade Católica do Rio Grande do Sul. Av. Ipiranga 6690, sala 220, Porto Alegre, RS 90610-000, Brasil. magdalahorgue@conex.com.br nunes@pucrs.br jcc@pucrs.br maurer@via-rs.net

2 Department of Paediatrics, The Chinese University of Hong Kong 6/F, Clinical Sciences Building, Prince of Wales Hospital, Shatin NT, Hong Kong, China. tony-nelson@cuhk.edu.hk

3 Education for Change Ltd 117 Clyde Road, PO Box 29170, Christchurch,

New Zealand.

scowan@efc.co.nz

4 Centro Latinoamericano de Perinatología

y Desarollo Humano.

Hospital de Clínicas, Piso 16, Casilla de Correo 627,

Montevideo 11000, Uruguay.

cafferam@clap.ops-oms.org

\begin{abstract}
The prone sleeping position has been causally linked to sudden infant death syndrome (SIDS). This survey aimed to determine whether maternity hospitals in Brazil were promoting the prone sleeping position for newborns. Information was al so collected on SIDS prevention messages. The study adopted a multicenter collaborative approach in hospitals with training in pediatrics, using questionnaires. Correctly completed questionnai res were received from 55 hospitals. The number of del iveries/year varied at the hospitals from 240 to 11,000 (median 2,750). The majority of hospitals encouraged the lateral (side) sleeping position in the normal care nurseries (44.4\%) and at discharge (67\%). In conclusion, the maj ority of hospitals surveyed are not promoting the supine sleeping position or any other intervention for SIDS prevention, although verbal information is provided to parents about breastfeeding (100\%), immunization (100\%), and smoking (85\%).
\end{abstract}

Key words Maternity Hospitals; Sudden Infant Death; Child Health; Sleep

Resumo A posi ção prona para dormir, tem si do relacionada como fator de risco para síndrome da morte súbita do lactente (SMSL). Com o objetivo de identificar qual a posi ção preferencial para dormir adotada nos hospitais, que ori entações são forneci das aos pais de recém-nascidos sobrea posi ção preferencial para dormir durante o primei ro ano de vida e prevenção de SMSL, realizamos estudo multicentrico por meio de questi onário, sendo sel ecionados hospitais com programa de Residência Médica em Pediatria. Anal isamos as informações referentes a 55 institui ções, cuja média de nasci mentos/ano variou entre 240 e 11.000 (mediana 2.750). O decúbito lateral é a posição preferencial para dormir (44,4\%) e a mais orientada para uso domiciliar (67\%). Concluímos que, a recomendação da posição supina ao dormir e orientações para prevenção da SMSL, não têm si do realizadas na rotina dos hospitais pesquisados. Entretanto, na alta hospitalar, o incentivo ao aleitamento materno e a importância das imunizações, são enfati zados em 100\% das insti tui ções e recomendações sobre evitar fumo, em 85\%.

Palavras-chave Maternidades; Morte Infantil Súbita; Saúde Infantil; Sono 


\section{Introdução}

A síndrome da morte súbita do lactente (SMSL), é a principal causa de mortalidade infantil nos países desenvolvidos, sendo considerada importante problema de saúde pública, já que é responsável por aproximadamente 10.000 mortes/ ano (Mitchell, 1990). Sua etiologia permanece desconhecida. Estudos populacionais, realizados na década de 80, identificaram fatores de risco dentro das práticas, até então usuais, do manejo domiciliar do lactente (Fleming et al., 1990; Mitchell et al., 1997; Ponsoby et al., 1993). A posição prona para dormir (também denominada frontal ou decúbito ventral) foi identificada como o principal fator de risco para SMSL (Malloy, 1998). Este estudo foi planejado baseado no fato de que o estabelecimento de práticas habituais para dormir, parece ser influenciado pelas orientações dadas à mãe do recém-nascido ainda no hospital. A hipótese inicial testada, é de que ainda existiriam países ou regiões promovendo a posição prona para dormir, e que conseqüentemente, teriam maior prevalência de SMSL, possivelmente subdiagnosticada.

\section{Métodos}

Esta pesquisa fez parte de estudo multicentrico internacional, coordenado pela Organização Mundial da Saúde (OMS - AMRO e EURO), SIDS (Sudden Infant Death Syndrome) Global Strategy e SIDS I nternational. Foi realizado estudo de corte transversal, descritivo, baseado em questionários aplicados em hospitais. O questionário foi elaborado pela OMS eSIDSInternational, apresentando oito perguntas fechadas: número de nascimentos/ ano da maternidade em questão, posição preferencial para dormir utilizada no berçário/alojamento conjunto e Unidade de Tratamento Intensivo (UTI) Neonatal, rotinas do berçário/alojamento conjunto (incluindo uso de cobertores, travesseiros, chupeta, tipo de colchão, número de agasalhos utilizados no recém-nascido, posição do (ençol), orientações verbais e impressas dadas aos pais de recém-nascidos antes da alta hospitalar. As perguntas sobre posição preferencial para dormir, tinham três respostas não excludentes: decúbito ventral, decúbito dorsal ou decúbito lateral (cada uma acompanhada de desenho ilustrativo) e as respostas para cada posição eram sempre, quase sempre, às vezes e nunca. No Brasil, os questionários foram aplicados em hospitais-escola para que as respostas pudessem ser representativas da insti- tuição. Foram incluídos hospitais com berçário ou cuja taxa de nascimento era superior a 200 partos/ano.

\section{Resultados}

Foram enviados questionários para 115 hospitais, entretanto, somente 55 retornaram e constituem a amostra analisada neste estudo. Estes hospitais correspondem a 37 cidades de 14 estados brasileiros (59\% da Região Sudeste, $14,5 \%$ das Regi ões Sul e Centro-oeste e $12 \%$ do Nordeste). O número de nascimentos/ano das maternidades incluídas, variou entre 240 e 11.000 (média $3.220 \pm 2.468$, mediana 2.750 ), com total de 137.230 nascimentos. Esse número representa $4 \%$ do total de nascimentos no Brasil em 1999.

A posição para dormir, preferencialmente utilizada no berçário/ alojamento conjunto, parece ser o decúbito lateral $(44,4 \%)$, seguida de decúbito dorsal $(24,1 \%)$ e decúbito ventral $(11,1 \%)$. Não foi definida posição preferencial em $5,6 \%$ dos hospitais e $14,8 \%$ enviaram dados inconsistentes. Na UTI Neonatal, a posição preferencial para dormir é o decúbito dorsal (37\%), seguido de ventral (17\%) e lateral (15\%). $11 \%$ dos hospitais não têm uma posição definida e $20 \%$ responderam de forma inconsistente. Nas orientações verbais dadas na alta hospitalar, $67 \%$ recomendam o decúbito lateral, $15 \%$ o dorsal, $5 \%$ o ventral e $13 \%$ não fazem qualquer recomendação neste aspecto. O sistema de alojamento conjunto, é utilizado em $91 \%$ das instituições, permanecendo os recém-nascidos no mesmo quarto com suas mães durante a noite, mas em seu próprio berço (89\%). A maioria dos recém-nascidos, dorme com 2 ou menos camadas de roupa sobre o tórax (70 \%), e são cobertos apenas abaixo do pescoço (61\%) com cobertor leve (65\%). 80\% são envolvidos frouxamente na coberta, permitindo que movimentem os braços. Somente $13 \%$ são colocados dentro de sacos de dormir. Sob o recémnascido, cobrindo o colchão, observa-se mais freqüentemente o uso exclusivo de lençol (98\%), seguido de forro plástico (35\%) ou cobertor de lã $(3,7 \%)$. Existe predomínio do uso de colchões intermediários (entre o firme e o macio) em $52 \%$, os demais usam tanto colchões moles $(20,4 \%)$ como duros $(26,4 \%)$. A maioria dos recém-natos (RN) dorme sem travesseiro (96\%), não usa chupeta (65\%) e não são colocados brinquedos dentro do berço na hora de dormir (85\%). As seguintes recomendações verbais são feitas de forma rotineira nos hospitais: incentivo ao aleitamento materno (100\%), imuniza- 
ções (100\%), evitar fumo na gestação (85\%), fumar próximo ao RN pode ser prejudicial (75\%).

\section{Discussão}

A SMSL representa significativo problema de saúde pública nos países desenvolvidos, onde é considerada a principal causa de mortalidade infantil. Após identificação da posição prona para dormir (decúbito ventral) como principal fator de risco para SMSL, campanhas de saúde pública advertindo pais e responsáveis para evitar a posição prona, resultaram em dramática redução, na ordem de 50\%, da mortalidade por SMSL; além disso, foi também verificado que a decisão dos pais de modificar a posição para dormir, é influenciada pela orientação recebida de profissionais da área de saúde (Fleming et al., 1990; Mitchell et al., 1997; Ponsoby et al., 1993).

Os resultados obtidos nos 55 hospitais brasileiros incluídos no estudo, demonstram que o decúbito lateral foi a posição preferencialmente adotada, tanto no alojamento conjunto como após a alta hospitalar, para recém-nascidos normais. Achados semelhantes, foram observados neste mesmo estudo, realizado em países do leste europeu (Nelson et al., 2000). Entretanto, como no Brasil o retorno de questionários foi inferior ao esperado, a generalização dos resultados fica comprometida. Apesar de toda informação disponível, observa-se que os profissionais da área da saúde ainda ficam receosos em modificar a orientação sobre posição ao dormir, pelo medo de que a posição supina possa aumentar o risco de complicações como aspiração de conteúdo gástrico. É admissível pensar que nos hospitais onde a posição supina nunca seja utilizada, exista, em verdade, pouco conhecimento sobre fatores de risco para SMSL. No Brasil, isto é facilmente compreensível, já que a inexistência de dados epidemiológicos sobre SMSL, faz com que os profissionais de saúde não consi derem esta como causa de mortalidade infantil importante, e conseqüentemente a prevenção não é realizada. A prevalência de SMSL é desconhecida em nosso país, exceto por duas cidades no Rio Grande do Sul: Pelotas e Porto Alegre. No estudo realizado em Porto Alegre, observamos que a SMSL é subdiagnosticada, mas mesmo assim, o coeficiente específico de mortalidade para SMSL é baixo (4,5:10.000 nascidos vivos), entretanto, em todos os casos detectados a posição usual de dormir era o decúbito lateral (Nunes et al., 2001). No estudo realizado em Pelotas, onde $4 \%$ dos óbitos no primeiro ano de vida foram possivelmente decorrentes de SMSL, não foram relatadas informações sobre posição de dormir (Barros et al., 1987). Apesar da posição supina não ser a mais utilizada, a posição preferencial recomendada, o decúbito lateral, traz um risco de SMSL inferior ao da posição prona. Maiores informações sobre prevalência de SMSL em nosso país, são necessárias para justificar o estabelecimento de campanhas para promoção da posição supina.

\section{Agradecimentos}

Agradecemos a todos os colegas que prontamente responderam aos questionários e possibilitaram a realização deste estudo. Dorothy Ford colaborou na montagem do questionário original em inglês, que foi inicialmente utilizado em Melbourne, Austrália. A Norwegian SIDS Society financiou o projeto. 


\section{Referências}

BARROS, F. C.; VICTORA, C. G.; VAUGHAN, J. P.; TEIXEIRA, A. M. B. \& ASHWORTH, A., 1987. Infant mortality in Southern Brazil: A population based study of causes of death. Archives of Disease in Childhood, 62:487-490.

FLEMING, P. J.; GILBERT, R.; AZAZ, Y.; BERRY, P. J.; RUDD, P. T.; STEWART, A. \& HALL, E., 1990. Interaction between bedding and sleeping position in the sudden infant death syndrome: A population based case-control study. BMJ, 301:85-89.

MALLOY, M. H., 1998. Effectively delivery in the message on infant sleep position. JAMA, 280:4.

MITCHELL, E. A., 1990. International trends in post neonatal mortality. Archives of Disease in Childhood, 65:607-609.

MITCHELL, E. A.; TUOHY, P. G.; BRUNT, J. M.; THOMPSON, J. M.; CLEMENTS, M. S.; STEWART, A. W.; FORD, R. P. \& TAYLOR, B. J., 1997. Risk factors for sudden infant death syndrome following the prevention campaign in New Zealand: A prospective study. Pediatrics, 100:835-840.
NELSON, E. A. S.; SERRA, A.; COWAN, S. \& MANGIATERRA, V., 2000. Maternity advice survey: Sleeping position in Eastern Europe. MAS Study Group for WHO EURO region. Archives of Disease in Childhood, 83:304-306.

NUNES, M. L.; PINHO, A. P. S.; AERTS, D.; SANT'ANNA, A.; M ARTINS, M. P. \& COSTA, J. C., 2001. Síndrome da morte súbita do lactente: Aspectos clínicos de uma doença subdiagnosticada. Jornal de Pediatria, 77:29-34.

PONSONBY, A. L.; DWYER, T.; GIBBONS, L. E. COCHRANE, J. A. \& WANG, Y. G., 1993. Factors potentiating the risk of sudden infant death syndrome associated with the prone position. New England Journal of Medicine, 329:377-382.

Recebido em 23 de julho de 2001

Versão final reapresentada em 28 de dezembro de 2001 Aprovado em 15 de janeiro de 2002 